\section{Dominant-Negative Mutants of a Toxin Subunit: An Approach to Therapy of Anthrax}

\author{
Bret R. Sellman, Michael Mourez, R. John Collier*
}

The protective antigen moiety of anthrax toxin translocates the toxin's enzymic moieties to the cytosol of mammalian cells by a mechanism that depends on its ability to heptamerize and insert into membranes. We identified dominantnegative mutants of protective antigen that co-assemble with the wild-type protein and block its ability to translocate the enzymic moieties across membranes. These mutants strongly inhibited toxin action in cell culture and in an animal intoxication model, suggesting that they could be useful in therapy of anthrax.

The increase of antibiotic resistance among pathogenic bacteria in recent years has prompted research to identify new approaches to treating bacterial infections. One approach is to develop ways to block the action of virulence factors. Toxic proteins are known to be important factors in many bacterial diseases, in that they are responsible for major symptoms (1), and for some diseases (e.g., diphtheria, tetanus, and pertussis) immunizing against a single toxic protein is known to provide protection against infection. Antibodies against toxins have sometimes been used to treat or prevent toxinrelated diseases, but toxins have generally not been targets for newly developed antibacterial agents. Recently a mutation in a subunit of VacA, a toxin from Helicobacter pylori, was shown to inhibit the action of the toxin in vitro (2). Here we describe mutant forms of a subunit of anthrax toxin that are potent inhibitors of toxin action in vitro and in vivo.

Spores of Bacillus anthracis, the etiologic agent of anthrax, infect herbivores most often but can also cause localized or systemic infections in humans (3). The most lethal form of the human disease, inhalational anthrax, is produced when spores in the lungs initiate a systemic infection; death almost inevitably occurs within a few days. Anthrax bacilli produce a set of three proteins, protective antigen (PA; $83 \mathrm{kD}$ ), lethal factor (LF; 90 $\mathrm{kD})$, and edema factor (EF; $89 \mathrm{kD})(3)$, which are known collectively as anthrax toxin (ATx). These proteins are nontoxic individually, but act in binary or ternary combinations to produce shock-like symptoms and death. LF and EF enzymically modify molecular targets within the cytosol, and PA transports them from the mammalian cell surface to that compartment. LF is a $\mathrm{Zn}^{2+}$-protease that

Department of Microbiology and Molecular Genetics, Harvard Medical School, Boston, MA 02115, USA.

*To whom correspondence should be addressed. Email: jcollier@hms.harvard.edu cleaves several mitogen-activated protein kinase kinases, kills macrophages, and causes death of the host (4-6). EF is a calmodulindependent adenylate cyclase that causes edema and impairs neutrophil function (7).

After their secretion from $B$. anthracis as monomeric proteins, $\mathrm{PA}, \mathrm{LF}$, and EF undergo self-assembly on the surface of mammalian cells to form toxic cell-bound complexes (Fig. 1). Initially, PA binds its receptor and is activated by furin-related proteases $(8,9)$. $\mathrm{PA}_{63}(63 \mathrm{kD})$, the activated form, spontaneously self-associates to form ring-shaped heptamers $(10,11)$, which bind LF and EF competitively and with high affinity $\left(K_{\mathrm{d}} \sim 1\right.$ $\mathrm{nM})(12,13)$. The resulting cell-associated complexes are endocytosed and trafficked to an acidic compartment (14), where the heptameric $\mathrm{PA}_{63}$ moiety inserts into the endosomal membrane and mediates translocation of EF and LF to the cytosol (15). Membrane insertion and pore formation by $\mathrm{PA}_{63}$ are believed to proceed via a heptameric preinsertion intermediate (prepore) (10) analagous to that of Staphylococcal $\alpha$-toxin (16). There is evidence that the prepore undergoes a conformational change under the influence of low $\mathrm{pH}$ that enables the $2 \beta_{2}-2 \beta_{3}$ loops (residues $302-325$ ) to come together and form a 14-strand $\beta$ barrel that spans the membrane $(10,17)$.

Mutations in PA that specifically block translocation are known, including a deletion of the $2 \beta_{2}-2 \beta_{3}$ loop (18) and point mutations in any of three residues, K397, D425, and F427 $(19,20)$. Because none of these mutations seriously impairs proteolytic activation of PA or self-assembly of the toxin, we reasoned that translocation-deficient PA mutants might co-assemble with wild-type (WT) PA to form hetero-heptamers. Further, we hypothesized that some of the mutants might be dominant negative (DN). Thus, incorporation of a small number of mutant subunits - perhaps only one-into an otherwise WT heptamer might impair translocation, making these proteins inhibitors of toxin action.

Translocation-deficient mutants. As an initial test of this hypothesis, we mixed various amounts of each of six mutant forms of PA with a constant amount of WT-PA and measured the ability of the mixtures to deliver a cytotoxic ligand, $\mathrm{LF}_{\mathrm{N}}$-DTA, into $\mathrm{CHO}$ $\mathrm{K} 1$ cells. $\mathrm{LF}_{\mathrm{N}}$-DTA, a fusion protein between $\mathrm{LF}_{\mathrm{N}}$, the PA-binding domain of LF, and DTA, the catalytic domain of diphtheria toxin (21), was used as a marker for PA-dependent translocation largely for convenience. It is translocated with about the same efficiency as LF and EF (22), and its entry into cells is easily quantified by monitoring protein synthesis. The DTA moiety catalyzes the adenosine diphosphate (ADP)-ribosylation of elongation factor-2 within the cytosol, inhibiting translation.

Of the translocation-deficient mutants tested, four manifested strong inhibitory activities: a double mutant, Lys ${ }^{397} \rightarrow \mathrm{Asp}^{397}$, $\mathrm{Asp}^{425} \rightarrow \mathrm{Lys}^{425}$ (K397D,D425K); a mutant with the $2 \beta_{2}-2 \beta_{3}$ loop deleted; and two point mutants, $\mathrm{Phe}^{427} \rightarrow \mathrm{Ala}^{427}(\mathrm{~F} 427 \mathrm{~A})$ and $\mathrm{D} 425 \mathrm{~K}$. The most potent member of this group, the double mutant, almost completely blocked toxin action at a 1:1 ratio of mutant: WT-PA. The deletion, F427A, and D425K mutants were only slightly less inhibitory. Another translocation-deficient mutant,

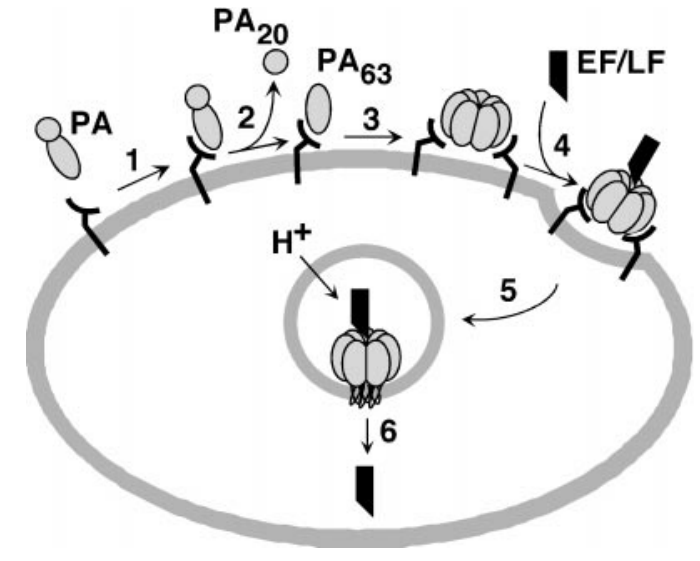

Fig. 1. Model of anthrax toxin action. 1) PA binds to cell surface receptor. 2) Furin-related proteases cleave PA, removing the amino terminal $20-\mathrm{kD}$ segment $\left(\mathrm{PA}_{20}\right)$. 3) $\mathrm{PA}_{63}$ oligomerizes to form the heptameric prepore. 4) LF and/ or EF binds to the prepore. 5) The prepore-LF and/or EF complex is taken up by receptor-mediated endocytosis and trafficked to the endosome. 6) Decrease in $\mathrm{pH}$ causes the prepore moiety to insert into endosomal membrane, form a pore, and translocate LF and/or EF to the cytosol. [Reproduced with permission from Biochemistry 38, 10432 (1999). Copyright 1999 American Chemical Society.] 


\section{RESEARCH ARTICLES}

K397D, caused virtually no inhibition at a $1: 1$ ratio, showing that not all mutants of this type are strongly inhibitory (Fig. 2). The remaining mutant, SSSR, contains a lesion that blocks activation by furin; it is unable to undergo oligomerization and ligand binding (23) but competes for PA receptors. The SSSR mutant caused no inhibition of toxin action, even in 10-fold excess over WT-PA, implying that competition for receptors did not contribute to the inhibitory activities of the other mutants. Thus, of the six mutants tested, four exhibited properties consistent with a DN phenotype.

Hybrid complex formation. The hypothesis that inhibition by the DN mutants depends on the ability of their $\mathrm{PA}_{63}$ moieties to form hybrid complexes with WT-PA 63 was tested using purified homo- and hetero-heptamers. PA in solution can be cleaved at the furin site by mild trypsinization, and the resulting fragments can be separated by chromatography of the trypsin-nicked molecule on an anion-exchange column (18). Purified $\mathrm{PA}_{63}$ isolated by this method is heptameric, indicating that the oligomerization equilibrium is greatly in favor of this form, and may be structurally similar or identical to the prepore. Purified homo-heptamers were prepared from WT-PA and each of five translocation-deficient PA mutants. Putative hetero-heptamers were prepared by mixing each mutant PA 1:1 with WT-PA, treating the mixture with trypsin, and performing chroma-

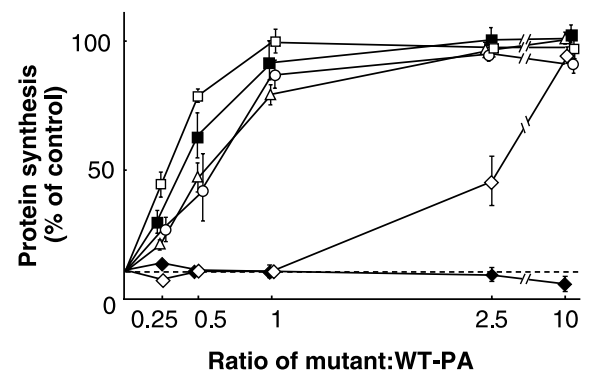

Fig. 2. Translocation-deficient PA mutants exhibit dominant-negative phenotype. CHO-K1 cells $\left(2.5 \times 10^{4}\right.$ cells/well) in a 96-well plate were incubated for 18 hours at $37^{\circ} \mathrm{C}$ with WTPA $(100 \mathrm{pM})$ in the presence of $\mathrm{LF}_{\mathrm{N}}$-DTA (100 PM) and various amounts of individual PA mutants [double $(\square)$, deletion ( $\square$ ), F427A $(\bigcirc)$, D425K $(\triangle)$, K397D $(\diamond)$, and SSSR $(\diamond)]$. The medium was then removed and replaced with leucine-free HAM F-12 supplemented with ${ }^{3} \mathrm{H}$ Leu at $1 \mu \mathrm{Ci} / \mathrm{ml}$. After incubation for 1 hour at $37^{\circ} \mathrm{C}$, the cells were washed with ice-cold phosphate-buffered saline (PBS) followed by icecold $10 \%$ trichloroacetic acid (TCA). The quantity of ${ }^{3} \mathrm{H}$-Leu incorporated into the TCA-precipitable material was measured and is expressed as percent of that incorporated in the absence of PA. At the concentrations of WT-PA and $\mathrm{LF}_{\mathrm{N}}$-DTA chosen, protein synthesis was inhibited by about $90 \%$ in the absence of mutant PA (dotted line). The mean of three experiments \pm SEM is reported. Similar results were seen when the initial incubation was 4 hours, instead of 18 hours (24). tography of the products on an anion-exchange column.

We measured the $\mathrm{LF}_{\mathrm{N}}$-DTA-dependent inhibition of protein synthesis by each hetero-heptamer and by an equivalent amount of a 1:1 mixture of the corresponding mutant and WT homo-heptamers. Hetero-heptamers containing the double mutant, the deletion, and the F427A and D425K mutants did not mediate the action of $\mathrm{LF}_{\mathrm{N}^{-}}{ }^{-}$ DTA, whereas the corresponding mixtures of homo-heptamers were highly active (Fig. 3). In contrast, with the K397D mutant the putative hetero-heptamer was as active as the homo-heptamer mixture. These results are consistent with the properties of these mutants in the experiment of Fig. 2 and support the notion that $\mathrm{PA}_{63}$ from the DN mutants inactivates the WT protein by co-oligomerizing with it. The absence of inhibitory activity of K397D in the hetero-heptamer preparation may reflect a defect either in ability to co-oligomerize with the WT protein or in ability to inhibit its activity within a heptamer. The finding that mutant homo-heptamers did not inhibit the activity of the WT indicates that little competition for receptors and little or no subunit exchange among heptamers occurred under the conditions of the experiment.

If co-oligomerization is purely stochastic,

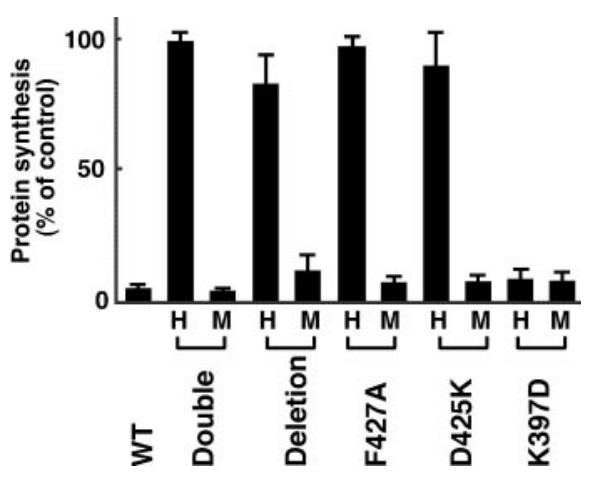

Fig. 3. Comparison of activities of hetero-heptamers of WT- and mutant PA with those of mixtures of the corresponding homo-heptamers. Homo-heptamers of WT-PA ${ }_{63}$ and the double, deletion, F427A, K397D, and D425K mutants were prepared as described (18). Putative hetero-heptamers were prepared by mixing each mutant PA with WT-PA in a 1:1 ratio before trypsinization and column chromatography. WT-PA (1 nM), hetero-heptamer $(\mathrm{H})$ (final concentration $2 \mathrm{nM}$ ), or an equimolar mixture (M) (1 nM each) of the corresponding mutant homo-heptamer and WT-heptamer was incubated with $\mathrm{CHO}-\mathrm{K} 1$ cells in the presence of LF $_{N^{-}}$-DTA (100 pM) for 18 hours, and inhibition of protein synthesis was measured as described in Fig. 2. Heptamer concentrations are expressed in terms of monomeric $\mathrm{PA}_{63}$ subunits. Protein synthesis is expressed as the percent of a control without PA. The mean of three experiments \pm SEM is reported. Similar results were seen after a 4 hour incubation (24). at a $1: 1$ ratio of mutant:WT-PA $\sim 0.8 \%$ of the heptamers formed would contain only WT subunits. Thus, if a single DN subunit is sufficient to inactivate a heptamer, one would expect $\sim 99 \%$ inhibition of activity at this ratio. The fact that the double mutant almost completely blocked activity in the two experiments described suggests both that a single molecule of the mutant inactivates a heptamer and that oligomerization is stochastic. The deletion, D425K, and F427A mutants appear to be slightly less inhibitory, implying that more than one molecule of these mutants per heptamer is required for inactivation and/ or that their co-oligomerization with WT-PA is not purely stochastic.

That the DN-PAs do in fact block the translocation activity of WT-PA was confirmed in a cell surface translocation assay (Fig. 4) (22). We bound trypsin-nicked PA to cells, added $\left[{ }^{35} \mathrm{~S}_{\mathrm{LF}} \mathrm{N}\right.$, and induced translocation of the $\mathrm{LF}_{\mathrm{N}}$ across the plasma membrane by acidifying the medium. Mixing an equal amount of a DN mutant with WT-PA before nicking did not diminish the amount of radiolabeled $\mathrm{LF}_{\mathrm{N}}$ that bound cells, but almost completely inhibited its translocation to the cytosol (Fig. 4). The SSSR mutant caused little

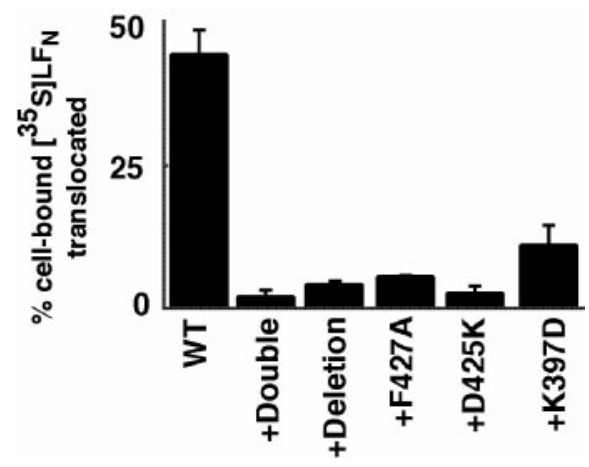

Fig. 4. Effect of dominant-negative PA mutants on low-pH triggered translocation of $\left[{ }^{35} \mathrm{~S}\right] \mathrm{LF}_{\mathrm{N}}$ across the plasma membrane. The cell-surface translocation assay described by Wesche et al. was employed (22). CHO-K1 cells were incubated for 2 hours on ice with PA $\left(2 \times 10^{-8} \mathrm{M}\right)$ in the presence or absence of an equal concentration of various mutants. The proteins were activated by incubation with trypsin before addition to the cells. The cells were then washed, incubated with $\left.{ }^{35} \mathrm{~S}\right] \mathrm{LF}_{\mathrm{N}}$ for another 2 hours, and washed again. The total cell-associated $\left[{ }^{35} \mathrm{~S}\right] \mathrm{LF}_{\mathrm{N}}$ was then determined by lysing the cells and measuring the radiolabel by scintillation counting. A parallel set of samples was incubated for $1 \mathrm{~min}$ at $37^{\circ} \mathrm{C}$ with $\mathrm{pH} 5.0$ buffer to trigger translocation. $\left[{ }^{35} \mathrm{~S}\right] \mathrm{LF}_{\mathrm{N}}$ remaining on the surface of the cell was digested with Pronase (Sigma, St. Louis, MO), and the cells were washed and lysed. The $\left[{ }^{35} \mathrm{~S}\right] \mathrm{LF}_{\mathrm{N}}$ translocated into the cells was measured by scintillation counting. Data are presented as percent of cell-associated label that became Pronase-resistant in cells treated at low $\mathrm{pH}$. Results presented are the mean of three experiments \pm SEM. 


\section{RESEARCH ARTICLES}

inhibition under these conditions (24).

Toxin inhibition in vivo. The properties displayed by the DN mutants in vitro imply that they should inhibit toxin action in vivo. To test this, we measured the activities of three of these mutants (the double, the deletion, and the F427A) in a classical in vivo model for ATx action, the Fisher 344 rat (25). We first confirmed that male rats ( 250 to $300 \mathrm{~g}$ ) injected intravenously with a mixture of $8 \mu \mathrm{g} \mathrm{LF}$ and 40 $\mu \mathrm{g}$ PA $(\approx 10$ times the minimal lethal dose) become moribund after $\sim 90$ min (Table 1). When we replaced PA in this mixture with any of the DN mutants, the animals showed no symptoms of intoxication (to the point of killing at 2 weeks). When we added DN-PA to the WT-PA/LF mixture before injection, either at a $1: 1$ ratio relative to WT-PA ( $40 \mu \mathrm{g}$ DN-PA) or a $0.25: 1$ ratio $(10 \mu \mathrm{g}$ DN-PA), the injected animals also survived without symptoms. The SSSR mutant had little effect on the activity of the toxin. These results are consistent with our in vitro results and demonstrate that the DN mutants can ablate anthrax toxin action in vivo, even at a sub-stoichiometric $(0.25: 1)$ ratio to WT-PA.

Conclusions. We have demonstrated that it is possible to convert a subunit of an oligomeric toxin into a potent inhibitor of toxin action by introducing any of several mutations. These mutations do not impair toxin assembly, but rather block an essential conformational transition of the assembled complex. These results, and those with another toxin system (2), underscore the possibility of identifying DN forms of a number of oligomeric virulence factors, ranging from toxins to adhesins.

In ATx and other oligomeric systems in which the assembly process occurs in contact with the extracellular milieu, exogenously added mutant subunits can in principle be incorpo- rated into the final structure, raising the possibility that such subunits could be used therapeutically. Systemic anthrax, although rare as a natural disease, is feared as an agent of biological warfare and terrorism, and DN-PA would seem to be a worthy candidate for a therapeutic. Assuming that administered DNPA intermixes freely with WT-PA produced in the body by $B$. anthracis, the proteins should co-assemble on cells to form inactive, dead-end complexes, thereby blocking the actions of both LF and EF. Our findings indicate that native proteins, but not the $\mathrm{PA}_{63}$ heptamers derived from them, could be effective inhibitors. Besides preventing overt symptoms, DN mutants may also protect macrophages and neutrophils from destruction, thereby aiding the host in eradicating the infection. No side effects have been observed following injection of WT-PA into humans, and thus a mutant inactive form of the protein should pose no hazard.

DN-PA may also be useful as a basis for a new vaccine against anthrax. As its name connotes, PA induces protective antibodies against anthrax, and indeed is the major immunogen of the vaccine currently licensed in the United States (26). We have found that the double, the deletion, and the F427A mutants exhibit little or no diminution in immunogenicity relative to WT-PA in Fisher rats (27). Purified WT-PA is under consideration as a replacement for the currently licensed vaccine (3), and if a DN form of PA proves efficacious therapeutically, it might fulfill this role as well, eliminating the need to develop two almost identical pharmaceuticals.

Note added in proof: Singh et al. report that a mutant PA different from those described here is dominant negative (28).

Table 1. Dominant negative PA mutants inhibit lethal toxin in rats. Male Fisher 344 rats ( 250 to $300 \mathrm{~g}$, Charles River Laboratories, Wilmington, MA) were injected intravenously with a mixture (final volume $200 \mu \mathrm{l})$ of $40 \mu \mathrm{g} \mathrm{WT}-\mathrm{PA}, 8 \mu \mathrm{g} \mathrm{LF}(25)$, and the quantities of mutants listed in the table. Before injection, the animals were anesthetized by intraperitoneal injection of ketamine $(1 \mathrm{mg})$ and acepromazine $(4 \mu \mathrm{g})$. Outcome is reported as time until moribund (TTM), defined as the point at which the symptoms of intoxication (extremely labored breathing and prostration) were such that the animal would not recover. At this point the animals were killed. Groups of four animals were used for each set of conditions, except for WT-PA alone, in which case a group of eight was used. Animals that survived exhibited no symptoms and were killed after 2 weeks.

\begin{tabular}{|c|c|c|c|c|c|}
\hline \multicolumn{5}{|c|}{ Quantity of protein $(\mu g)$} & \multirow{2}{*}{ TTM } \\
\hline WT & Deletion & Double & $\mathrm{F} 427 \mathrm{~A}$ & SSSR & \\
\hline 40 & - & - & - & - & $90 \pm 11 \mathrm{~min}$ \\
\hline- & 40 & - & - & - & Survived \\
\hline - & - & 40 & - & - & Survived \\
\hline- & - & - & 40 & - & Survived \\
\hline 40 & 40 & - & - & - & Survived \\
\hline 40 & - & 40 & - & - & Survived \\
\hline 40 & - & - & 40 & - & Survived \\
\hline 40 & - & - & - & 40 & $100 \pm 3 \min$ \\
\hline 40 & 10 & - & - & - & Survived \\
\hline 40 & & 10 & - & - & Survived \\
\hline 40 & - & - & 10 & - & Survived \\
\hline
\end{tabular}

References and Notes

1. R. Rapuoli, M. Pizza, in Cellular Microbiology P. Cossart, P. Boquet, S. Normark, R. Rappuoli, Eds. (American Society for Microbiology Press, Washington, DC, 2000), pp. 193-220.

2. A. D. Vinion-Dubiel et al., J. Biol. Chem. 274, 37736 (1999).

3. T. C. Dixon, M. Meselson, J. Guillemin, P. C. Hanna, N. Engl. J. Med. 341, 815 (1999).

4. N. S. Duesbery et al., Science 280, 734 (1998).

5. G. Vitale et al., Biochem. Biophys. Res. Commun. 248 706 (1998).

6. G. Vitale, L. Bernardi, G. Napolitani, M. Mock, C. Montecucco, Biochem. J. 352, 739 (2000).

7. J. O'Brien, A. Friedlander, T. Dreier, J. Ezzell, S. Leppla, Infect. Immun. 47, 306 (1985).

8. V. Escuyer, R. J. Collier, Infect. Immun. 59, 3381 (1991)

9. K. R. Klimpel, S. S. Molloy, G. Thomas, S. H. Leppla, Proc. Natl. Acad. Sci. U.S.A. 89, 10277 (1992).

10. C. Petosa, R. J. Collier, K. R. Klimpel, S. H. Leppla, R. C. Liddington, Nature 385, 833 (1997).

11. J. C. Milne, D. Furlong, P. C. Hanna, J. S. Wall, R. J. Collier, J. Biol. Chem. 269, 20607 (1994).

12. S. H. Leppla, in Sourcebook of Bacterial Protein Toxins J. Alouf, Ed. (Academic Press, New York, 1991), pp. 277-302.

13. J. L. Elliott, J. Mogridge, R. J. Collier, Biochemistry 39, 6706 (2000).

14. A. M. Friedlander, J. Biol. Chem. 261, 7123 (1986).

15. T. M. Koehler, R. J. Collier, Mol. Microbiol. 5, 1501 (1991).

16. B. Walker, M. Krishnasastry, L. Zorn, H. Bayley, J. Biol. Chem. 267, 21782 (1992).

17. E. L. Benson, P. D. Huynh, A. Finkelstein, R. J. Collier, Biochemistry 37, 3941 (1998).

18. C. J. Miller, J. L. Elliott, R. J. Collier, Biochemistry 38, 10432 (1999).

19. B. R. Sellman, S. Nassi, R. J. Collier, J. Biol. Chem. 276, 8371 (2001).

20. Single-letter abbreviations for the amino acid residues are as follows: A, Ala; C, Cys; D, Asp; E, Glu; F, Phe; G, Gly; H, His; I, Ile; K, Lys; L, Leu; M, Met; N, Asn; P, Pro; Q, Gln; R, Arg; S, Ser; T, Thr; V, Val; W, Trp; and Y, Tyr.

21. J. C. Milne, S. R. Blanke, P. C. Hanna, R. J. Collier, Mol. Microbiol. 15, 661 (1995).

22. J. Wesche, J. L. Elliott, P. O. Falnes, S. Olsnes, R. J. Collier, Biochemistry 37, 15737 (1998).

23. K. E. Beauregard, R. J. Collier, J. A. Swanson, Cell. Microbiol. 2, 251 (2000).

24. B. R. Sellman, M. Mourez, R. J. Collier, data not shown.

25. B. E. Ivins, J. D. Ristroph, G. O. Nelson, Appl. Environ. Microbiol. 55, 2098 (1989).

26. A. M. Friedlander, P. R. Pittman, G. W. Parker, JAMA 282, 2104 (1999).

27. PA administered with Ribi Tri-Mix adjuvant elicits an anti-PA response and protects animals from challenge by $B$. anthracis spores (29). We tested the immunogenicity of the double, deletion, and F427A mutants, by vaccinating groups of six male Fisher 344 rats intramuscularly at 0,3 , and 6 weeks with each protein $(50 \mu g)$ mixed with the Ribi Tri-Mix adjuvant (Sigma) in PBS. The animals were bled prior to the first injection and 14 days following each injection. On day 16 after the final injection the animals were challenged by intravenous injection of a lethal dose of WT-PA plus LF (30 $\mu g+6 \mu g$, respectively). Mock-vaccinated animals became moribund 74 min after injection, whereas animals vaccinated with any of the DN mutants or with WT-PA survived without symptoms. Titers of PA-reactive and neutralizing antibodies elicited by the DN mutants (measured after the final injection) were greater than or equal to those elicited by WT-PA.

28. Y. Singh, H. Kanna, A. P. Chopra, V. Mehra, J. Biol. Chem., in press.

29. B. W. McBride et al., Vaccine 16, 810 (1998).

30. Supported by grant R37-AI22021 (R.J.C.) and training grant 5T32AI07410 (B.R.S.) from the NIH, DSP/DGA (9860830006) (M.M.), and the Fondation Philippe (M.M.). R.J.C. has financial interest in AVANT Immunotherapeutics, Inc.

5 February 2001; accepted 19 March 2001 